\title{
Effect of contact angle and helix angle on slide-roll ratio under the uniform motion state of BSM
}

\author{
Kong Deshun, Wang Min and Gao Xiangsheng \\ Key Laboratory of Advanced Manufacturing Technology, Beijing University of Technology, Beijing 100124, China
}

\begin{abstract}
To study the effect of contact and helix angles on slide-roll ratio at the ball contact point under the uniform motion state of ball screw mechanism (BSM), a slide- roll ratio model was derived in the literature [9] were used in this paper. It also conducts a simulation analysis of the slide-roll ratio relationship between the uniform motion at the ball center and ball contact point under different contact angle and helix angle. As shown by the analysis, with the increase in the BSM's contact angle, the slide/roll ratio at the contact point decreased, and the contact angle had a relatively significant effect on the slide/roll ratio; however, with the decrease in the BSM's helix angle, the slide/roll ratio at the contact point decreased, and helix angle had a relatively insignificant effect on the slide/roll ratio.
\end{abstract}

\section{Introduction}

As a key component of a numerical control machine tool, the performance of BSM has great impacts on the processing quality of the workplaces. During the numerous parameters of the BSM, it has been found that the running status between the balls and raceway has more significance influence on the performance of the BSM. The running status is restricted to the friction torque between the nut and the screw, contact angle, helix angle between the ball and raceway, and the preload, etc. Therefore, it is very important to affect the wear and precision retaining ability of the BSM. Wei [1-3] introduced the theory of differential geometry of curve to analyze the motion rules of a ball considering the effect of the elastic deformation of contact angle and found that the self-spin and rotational angular velocities of the ball have an approximately linear relationship with the rotational velocity of the screw. Lin et al. [4-5] confirmed the existence of both the rolling and sliding motions relative to each other between the ball and raceway during the operation of BSM and proposed a calculation model to estimate the transmission efficiency. Gnanamoorthy and Govindarajan [6-7] identified the slide/roll ratio of the contact point as the key factor influencing the wear velocity of the contact area. Ma [8] affirmed the influencing rules of the contact and helix angles of the BSM on the slide-roll ratio and found that both contact angle and helix angles had an opposite trend of the slide-roll ratio and an increase in helix angle significantly decreased the slide/roll ratio of the nut contact point. Li [9] established the model of the dynamic contact angle of BSM combined with the geometric parameters of the ball and the raceway between nut and screw. He verified that the inner contact angles in screw raceway are always greater than the external angles in nut raceway under high velocity working conditions. $\mathrm{Hu}[10]$ found five different motion states between the ball, the screw of raceways, and the nut of raceways by analysis and also evaluated the effects of the motion velocity of the ball on the slide-roll ratio. In the above cases, the motion rules of the ball were explored when the BSM is under a uniform motion and quasistatic state. However, there is no quantitative analysis about contact angle and helix angle of BSM. The motion characteristics of the ball during the uniform motion of the BSM decreased the wear of the BSM during this process and increased its precision and service life.

In this study, the influence of contact angle and helix angle on the slide-roll ratio is analyzed on the basis of $\mathrm{Hu}$ studied. It was found that an increase in contact angle can decrease the slide/roll ratio of the contact point; in contrast, a decrease in helix angle can decrease the slide/roll ratio of the contact point.

\section{Contact point position of ball}

The contact angle of the ball screw determines the relative positional relationship between the ball and the screw as well as the nut can be showed in Fig. 1. The position vectors of contact point $A$ between the ball and the screw of raceway and contact point $\mathrm{B}$ between the ball and the nut of raceway in the Frenet-Serret coordinate system $O_{\mathrm{H}} t n b$ is as follows:

$$
\boldsymbol{P}_{\mathrm{A}}^{\mathrm{H}}=\left[\begin{array}{c}
0 \\
r_{\mathrm{b}} \cos \beta_{\mathrm{A}} \\
r_{\mathrm{b}} \sin \beta_{\mathrm{A}} \\
1
\end{array}\right]
$$




$$
\boldsymbol{P}_{\mathrm{B}}^{\mathrm{H}}=\left[\begin{array}{c}
0 \\
-r_{\mathrm{b}} \cos \beta_{\mathrm{B}} \\
-r_{\mathrm{b}} \sin \beta_{\mathrm{B}} \\
1
\end{array}\right]
$$

where $\beta_{\mathrm{A}}$ is contact angle between the ball and the screw of raceway, $\beta_{\mathrm{B}}$ is contact angle between the ball and the nut of raceway, and $r_{\mathrm{b}}$ is the radius of the ball.

Taking the right-hand screw as an example for the convenience of studying the BSM, the nut moves along the negative direction of $Z_{\mathrm{W}}$ axis when the screw rotates around the positive direction of $Z_{\mathrm{W}}$ axis, and contact angles $\beta_{\mathrm{A}}$ and $\beta_{\mathrm{B}}$ are positive.

\section{Analysis of slide-roll ratio of BSM}

There were probably have slide-roll ratio at the contact point $A$ between the ball and the screw of raceway and contact point $\mathrm{B}$ between the ball and the nut of raceway, it can be obtained as [10]. The slide-roll ratio at contact point $\mathrm{A}$ and $\mathrm{B}$ can be written as:

$$
\begin{aligned}
& S_{A}= a b s\left(g \dot{\Omega}\left(\frac{r}{\cos \alpha}-r_{b} \cos \beta_{0} \cos \alpha\right)\right) /(a b s(g \dot{\Omega} \\
&\left.\left(\frac{r}{\cos \alpha}-r_{b} \cos \beta_{0} \cos \alpha\right)+\dot{\Omega} \cos \alpha\left(r-r_{b} \cos \beta_{0}\right)\right) \\
&\left.+a b s\left(\dot{\Omega} \cos \alpha\left(r-r_{b} \cos \beta_{0}\right)\right)\right) \\
& S_{B}=a b s\left(\left(2 v_{b t}-g \dot{\Omega}\left(\frac{r}{\cos \alpha}-r_{b} \cos \beta_{0} \cos \alpha\right)\right.\right. \\
&\left.+\dot{\Omega}\left(\frac{r}{\cos \alpha}+r_{b} \cos \beta_{B} \cos \alpha\right)\right) /(\operatorname{abs}( \\
&\left.2 v_{b t}-g \dot{\Omega}\left(\frac{r}{\cos \alpha}-r_{b} \cos \beta_{0} \cos \alpha\right)\right)+ \\
& a b s\left(-\dot{\Omega}\left(\frac{r}{\cos \alpha}+r_{b} \cos \beta_{B} \cos \alpha\right)\right)
\end{aligned}
$$

In the BSM, the screw drives the ball which further drives the nut at the same time, therefore, the velocity of the ball at contact point $\mathrm{A}$ in the tangential direction $t$ is less than that of the screw. Based on the relative velocity relationship expression

$$
\left[\boldsymbol{v}_{A S}^{H}\right]=\left[\begin{array}{c}
v_{b t}+\omega_{b n} r_{b} \sin \beta_{A}-\omega_{b b} r_{b} \cos \beta_{A} \\
-\omega_{b t} r_{b} \sin \beta_{A} \\
\omega_{b t} r_{b} \cos \beta_{A}
\end{array}\right] \text { of contact }
$$

point A between the ball and the screw of raceways on the relative velocity relationship expression $\boldsymbol{v}_{B S}^{H}=\left[\begin{array}{c}v_{b t}-\omega_{b n} r_{b} \sin \beta_{B}+\omega_{b b} r_{b} \cos \beta_{B} \\ +\dot{\Omega}\left(\frac{r}{\cos \alpha}+r_{b} \cos \beta_{B} \cos \alpha\right) \\ \omega_{b t} r_{b} \sin \beta_{B}+\dot{\Omega} r_{b} \sin \alpha \sin \beta_{B} \\ -\omega_{b t} r_{b} \cos \beta_{B}-\dot{\Omega} r_{b} \sin \alpha \cos \beta_{B}\end{array}\right]$ of contact point

$\mathrm{B}$ between the ball and the nut of raceways,

$$
\begin{aligned}
& -\dot{\Omega}\left(\frac{r}{\cos \alpha}+r_{b} \cos \beta_{B} \cos \alpha\right) \leq \\
& v_{b t}-\omega_{b n} r_{b} \sin \beta_{B}+\omega_{b b} r_{b} \cos \beta_{B} \leq 0
\end{aligned}
$$

Considering the two ultimate uniform motion states of the ball, i.e., it is motionless relative to the nut $v_{b t}=-\dot{\Omega} \frac{r}{\cos \alpha}$ and screw $\left\{\begin{array}{l}v_{b t}=0 \\ \omega_{b t}=\omega_{b n}=\omega_{b b}=0\end{array}\right.$, the velocity range of the ball along helix of the screw is:

$$
-\dot{\Omega} \frac{r}{\cos \alpha} \leq v_{b t} \leq 0
$$

Similarly, the linear velocity range of the ball in the tangential direction of helix path can be obtained using $\boldsymbol{v}_{A S}^{H}=\left[\begin{array}{c}v_{b t}+\omega_{b n} r_{b} \sin \beta_{A}-\omega_{b b} r_{b} \cos \beta_{A} \\ -\omega_{b t} r_{b} \sin \beta_{A} \\ \omega_{b t} r_{b} \cos \beta_{A}\end{array}\right]=\left[\begin{array}{l}0 \\ 0 \\ 0\end{array}\right]$
$\left\{\begin{array}{l}v_{b t}=\omega_{b b} r_{b} \cos \beta_{A}-\omega_{b n} r_{b} \sin \beta_{A} \\ \omega_{b t}=0\end{array}\right.$ as follows: and

$$
-\dot{\Omega} \sin \alpha \leq \omega_{b t} \leq 0
$$

Assuming that

$$
\begin{aligned}
\omega_{b n} r_{b} & \sin \beta_{0}=-v_{b t}+\omega_{b b} r_{b} \cos \beta_{0} \\
+g & \dot{\Omega}\left(\frac{r}{\cos \alpha}-r_{b} \cos \beta_{0} \cos \alpha\right)
\end{aligned}
$$

where $-1 \leq g \leq 0$,

The slide-roll ratio of the ball contact point $\mathrm{A}$ and $\mathrm{B}$ becomes:

$$
\begin{aligned}
& S_{A}= g \dot{\Omega}\left(\frac{r}{\cos \alpha}-r_{b} \cos \beta_{0} \cos \alpha\right) /(a b s(g \dot{\Omega} \\
&\left.\left(\frac{r}{\cos \alpha}-r_{b} \cos \beta_{0} \cos \alpha\right)+\dot{\Omega} \cos \alpha\left(r-r_{b} \cos \beta_{0}\right)\right) \\
&\left.+a b s\left(\dot{\Omega} \cos \alpha\left(r-r_{b} \cos \beta_{0}\right)\right)\right) \\
& S_{B}=\left(\left(2 v_{b t}-g \dot{\Omega}\left(\frac{r}{\cos \alpha}-r_{b} \cos \beta_{0} \cos \alpha\right)\right.\right. \\
&\left.+\dot{\Omega}\left(\frac{r}{\cos \alpha}+r_{b} \cos \beta_{B} \cos \alpha\right)\right) /(\mathrm{abs}( \\
&\left.2 v_{b t}-g \dot{\Omega}\left(\frac{r}{\cos \alpha}-r_{b} \cos \beta_{0} \cos \alpha\right)\right)+ \\
& a b s\left(-\dot{\Omega}\left(\frac{r}{\cos \alpha}+r_{b} \cos \beta_{B} \cos \alpha\right)\right)
\end{aligned}
$$

Based on a comprehensive consideration of Eq. (6), $g$ should also satisfy the following condition: 


$$
g \geq \frac{v_{\mathrm{bt}}^{*}-\omega_{\mathrm{bb}} r_{\mathrm{b}} \cos \beta}{\ddot{\Omega}\left(\frac{r}{\cos \alpha}-r_{\mathrm{b}} \cos \beta \cos \alpha\right)}
$$

By substituting the parameters shown in Table 1 into Eqs. (9) and (10) to arrive at the values satisfying Eqs.(7) and (8) and $-1 \leq g \leq 0$, the relationship between $v_{\mathrm{bt}}$ and $\omega_{\text {bn }}$ (Fig. 2) as well as those between slide-roll ratio and $v_{\text {bt }}\left(\omega_{\text {bn }}\right)($ Fig. 3/Fig. 4) can be worked out.

Table 1. Parameters of the BSM.

\begin{tabular}{lcc}
\hline \multicolumn{1}{c}{ Parameters } & Value & Unit \\
\hline Pitch radius $r$ & 16 & $\mathrm{~mm}$ \\
Ball radius $r_{b}$ & 2.9765 & $\mathrm{~mm}$ \\
Helix angle $\alpha$ & 5.6833 & degree \\
Contact angle $\beta_{0}$ & 44.5 & degree \\
Pitch $L$ & 5 & $\mathrm{~mm}$ \\
Screw angular velocity $\dot{\Omega}$ & $0-196$ & $\mathrm{rad} / \mathrm{s}$ \\
\hline
\end{tabular}

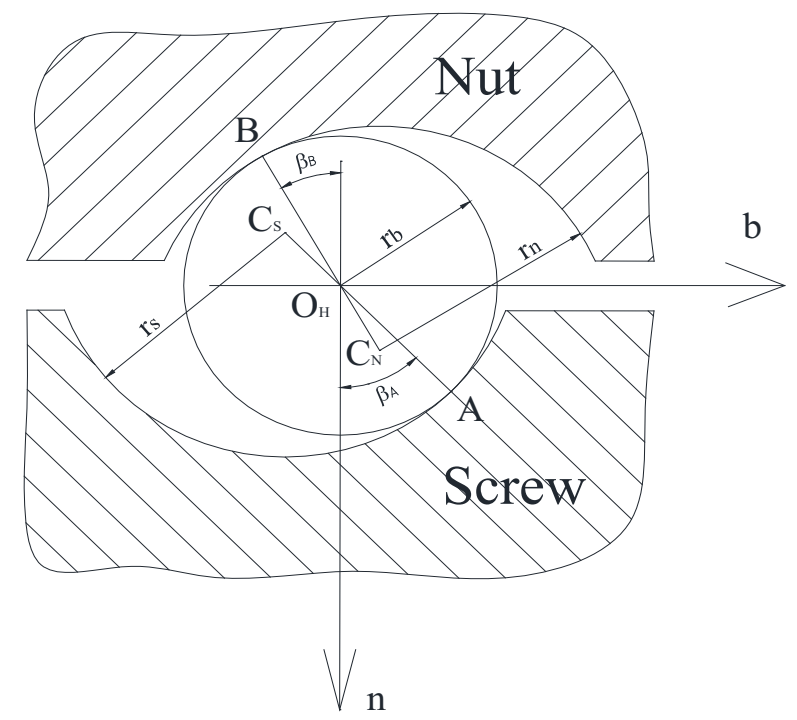

Figure 1. Contact angle of BSM.

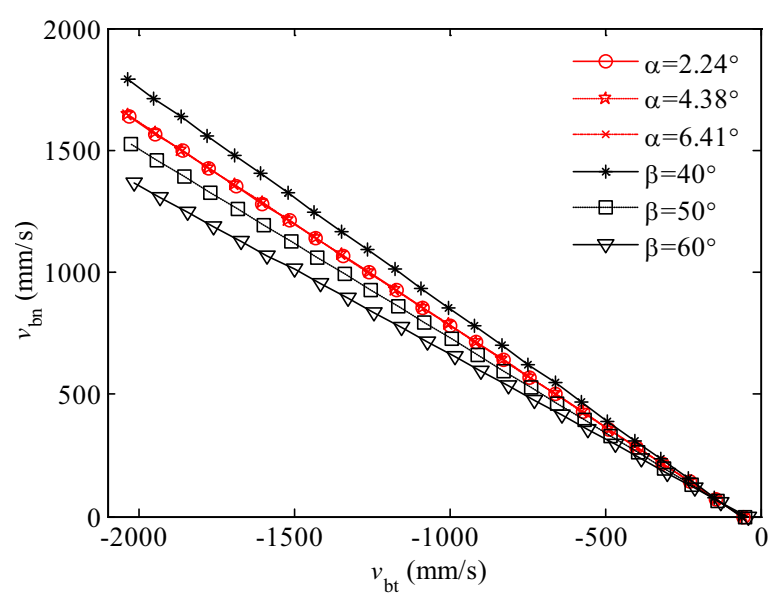

Figure 2. Relationship between $v_{\mathrm{bt}}$ and $\omega_{\mathrm{bn}}$.

\section{Results and discussion}

(1) When the BSM is under the same velocity, the greater the contact angles, the smaller value of $\omega_{\mathrm{bn}} / \nu_{\mathrm{bt}}$ in Fig.2; under the same velocity, the greater helix angles, the bigger value of $\omega_{\mathrm{bn}} / v_{\mathrm{bt}}$, but their values are affected by helix angle to a lesser degree. At the same time, with the increase in the value of $v_{\mathrm{bt}}, \omega_{\mathrm{bn}} / v_{\mathrm{bt}}$ changes with the permanent amplitude under the constant contact angle and helix angle.

(2) Under the same $v_{\mathrm{bt}}, \mathrm{S}_{\mathrm{A}}$ and $S_{\mathrm{B}}$ decrease with the increase in contact angle as shown in Fig. 3. Under the same $v_{\mathrm{bt}}, S_{\mathrm{A}}$ and $S_{\mathrm{B}}$ increase with the increase in helix angle, but their values are affected by helix angle to a lesser degree. Increasing the value of $v_{\mathrm{bt}}, S_{\mathrm{B}}$ changes with the smaller amplitude.

(3) As Fig. 4 shown, under the same $\omega_{\text {bn }}$, the greater contact angles, the bigger values of $S_{\mathrm{A}}$ first and the smaller later, but the decrease range is bigger. The greater contact angles, the smaller values of $S_{\mathrm{B}}$. The greater helix angles, the smaller values of $S_{\mathrm{A}}$ and the bigger values of $S_{\mathrm{B}}$, and with the variety of helix angles, the range of $S_{\mathrm{A}}$ and $S_{\mathrm{B}}$ are the smaller. At the same time, with the increase in $\omega_{\text {bn }}, S_{\mathrm{A}}$ and $S_{\mathrm{B}}$ each present the same change trend as under the influence of $v_{\mathrm{bt}}$.

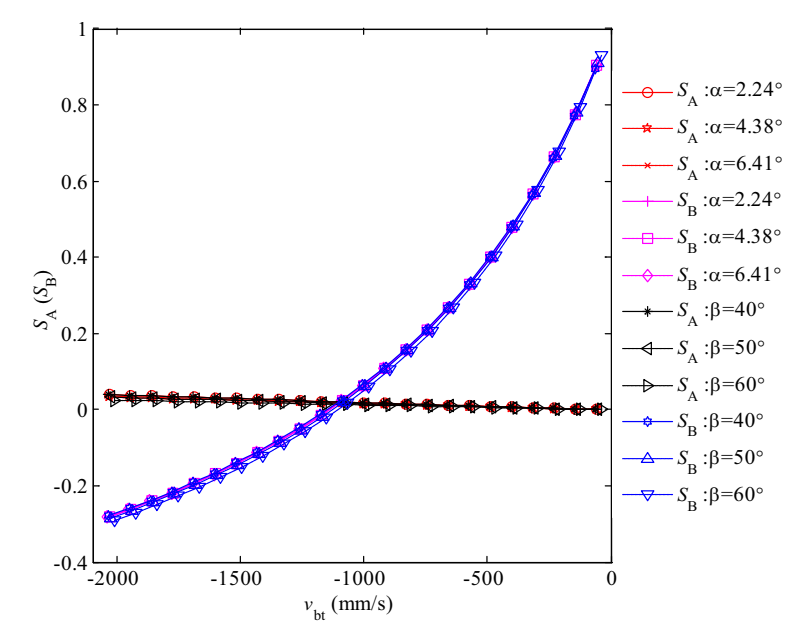

Figure 3. Relationship between $S_{\mathrm{A}}\left(S_{\mathrm{B}}\right)$ and $v_{\mathrm{bt}}$.

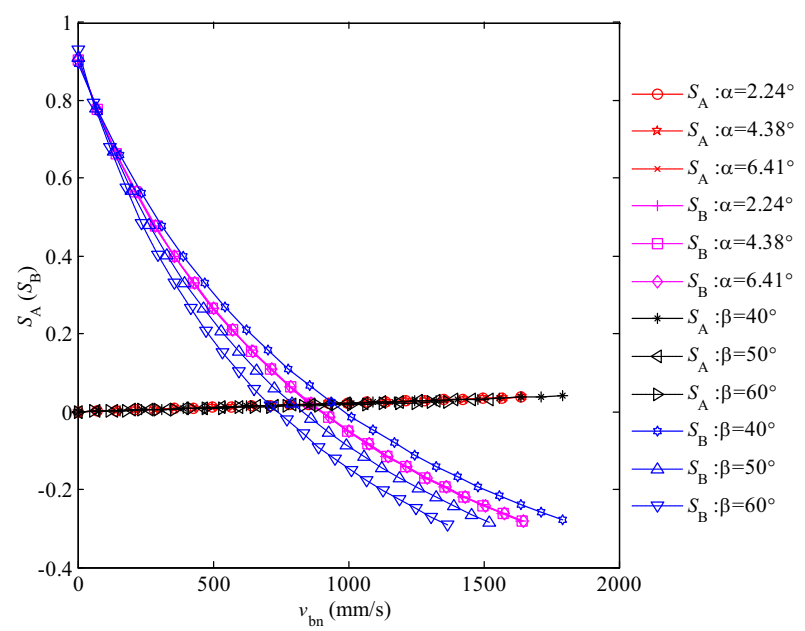

Figure 4. Relationship between $S_{\mathrm{A}}\left(S_{\mathrm{B}}\right)$ and $\omega_{\text {bn }}$ 


\section{Conclusions}

(1) Relationship between $\omega_{\mathrm{bn}}$ and $v_{\mathrm{bt}}$ of the contact points of BSM are analyzed, it also explores the effects of contact angle and helix angle on $\omega_{\mathrm{bn}}$ and $v_{\mathrm{bt}}$.

(2) Relationship between $S_{\mathrm{A}}$ and $S_{\mathrm{B}}, \omega_{\mathrm{bn}}$ and $v_{\mathrm{bt}}$ in the course of motion of the BSM are analyzed, at the same time, the effects of contact angle and helix angle on $S_{\mathrm{A}}$ and $S_{\mathrm{B}}$ are also explored.

\section{Acknowledgements}

This work is supported by National Natural Science Foundation of China, Grant No. 51575014 and 51505012, and Natural Science Foundation of Beijing, Grant No. KZ201410005010. Beijing Postdoctoral Research Foundation, Grant No. 2015XX-13. The authors are grateful to other participants of the projects for their cooperation.

\section{References}

1. Wei C C, Lin J F. Kinematic analysis of the ball screw mechanism considering variable contact angles and elastic deformations[J]. ASME Journal of Mechanical Design, 2003, 125: 717-733

2. Wei C C, Lin J F, Horng J H. Analysis of a ball screw with a preload and lubrication[J]. Tribology International, 2009, 42: 1816-1831
3. Wei C C, Lin J F, Horng J H. Kinematical analyses and transmission efficiency of a preloaded ball screw operating at high rotational velocitys $[\mathrm{J}]$. Mechanism and Machine Theory, 2011, 46: 880-898.

4. Lin M C, Ravani B, Velinsky S A. Kinematics of the ball screw mechanism[J]. ASME Journal of Mechanical Design, 1994, 116(3): 849-855

5. Lin M C, Ravani B, Velinsky S A. Design of the ball screw mechanism for optimal efficiency[J]. ASME Journal of Mechanical Design, 1994, 116(3): 856861

6. Gnanamoorthy R, Govindarajan N. Effect of slideroll ratio on the contact fatigue behavior of sintered and hardened steels[J]. Journal of Failure Analysis and Prevention, 2004, 4(2): 78-83

7. Govindarajan N, Gnanamoorthy R. Rolling/sliding contact fatigue life prediction of sintered and hardened steels[J]. Wear, 2007, 262: 70-78

8. Ma S J,Liu Geng,Fu Xxiaojun,et al. Rolling-sliding characteristics of planetary roller screw considering elastic deformation. Journal of Southeast University(Natural Sciences Edition),2015,45(3):461-468.

9. Li M Q,Yu H,Li D Y,et al. Modeling for dynamic contact angle of ball screw mechanism aimed to structural parameters. Transactions of the Chinese Society of Agricultural Engineering, 2016, 32(4):98104.

10. Hu J Z, Wang M, Zan T, The kinematics of ballscrew mechanisms via the slide-roll ratio, Mech. Mach. Theory 79 (2014) 158-172. 